\title{
Evolutionary Service-Oriented Architecture for Network Enabled Capability
}

\author{
Lu Liu, Duncan Russell, Nik Looker, David Webster \& Jie Xu \\ School of Computing, University of Leeds, Leeds, West Yorkshire, U.K. \\ \{luliu, duncanr, nlooker, dwebster\& jxu\}@comp.leeds.ac.uk \\ John K Davies \& Ken Irvin \\ BAE Systems, Integrated System Technologies, Victory Point, Frimley, U.K. \\ \{john.k.davies \& ken.irvin\}@baesystems.com
}

\begin{abstract}
The U.K. Ministry of Defence (MoD) aims to significantly enhance military effect through the networking of existing and future military capabilities, under the banner of Network Enabled Capability (NEC). To respond to this need, the EPSRC and BAE Systems are jointly funding the Network Enabled Capability Though Innovative Systems Engineering (NECTISE) project, which involves ten U.K. universities and is addressing the question of how BAE Systems delivers elements that contribute to NEC for its customers. One of the objectives of the NECTISE project is to develop a systematic approach that would lead to flexible service-oriented architectures for through-life evolution by investigating how loosely coupled services can be used to describe the functions and quality of service for heterogeneous systems and networks. In this paper, we present the concept of evolutionary service-oriented architecture (SOA) for NEC using agile methodologies to adapt to changes for the provision of dependable and sustainable military capability.
\end{abstract}

KEYWORDS : Network Enabled Capability, Agility, Service-Oriented Architecture

\section{INTRODUCTION}

The importance of achieving enhanced military effect through networking and system integration has been widely recognised by defence communities [1, 2]. Network Enabled Capability (NEC) is the U.K. Ministry of Defence (MoD)'s response to the quickly changing conflict environment in which its forces must operate. NEC offers decisive advantage through the timely provision and exploitation of information and intelligence to enable effective decision-making and agile actions [3]. 
To be successful in achieving this goal, the respective roles of U.K. government and industry in support of military capability are undergoing major changes at the same time as progress is made towards NEC aspirations. It is clear that provision of NEC must consider not only the networking of sensors and decision makers for military effect but how such a capability can be deployed, supported through-life, and used in a new defence acquisition paradigm in which the relationship between MoD and industry is changing [4].

The achievement of NEC has been set as the highest priority for the Advice to Capability Management research output, as well as being a strategic research priority for MoD [5]. To respond to this need, the U.K. Engineering and Physical Sciences Research Council (EPSRC) and BAE Systems are jointly funding the Network Enabled Capability Though Innovative System Engineering (NECTISE) project which is addressing the question of how BAE Systems delivers elements that contribute to NEC for its customers, taking account of the aims summarised in the 2005 Defence Industrial Strategy [6]. The University of Leeds is leading research activities on system architectures and contributing to the development of through life system management. Architectures for Network Enabled Capability and through-life provision of military capability are two challenges highlighted in the NECTISE project [4]. In this paper, we present the concept of evolutionary service-oriented architecture (SOA) for NEC using agile methodologies to adapt to changes for the provision of dependable and sustainable military capability.

This paper is organised as follows: Section 2 discusses part of an investigation into SOA for the support of dynamic military environments and delivery of NEC. Section 3 discusses agility in networking. A survey of relevant literature on complex networks is given by highlighting the characteristics of complex networks. Networking on complex networks is then discussed with regard to network connectivity and network awareness. Section 4 explores service-oriented architecture for the provision of military capability. In Section 5, conclusions are given and future work is outlined.

\section{SERVICE-ORIENTED ARCHITECTURE}

The use of service-oriented architecture has been motivated by many industries changing the focus from product delivery to service-oriented delivery. The focus on service delivery has also been apparent in software, where networking has become faster, more reliable and more available as a result of reduced cost. The SOA approach in software is used as an enabler for business process integration that characterises business functions as services, and integrates dynamically across departments and organisations [7-9].

The conceptual service-oriented architecture can be used to integrate businesses, systems and computing by using different levels of abstraction. The architecture is made of service suppliers and consumers, with suppliers advertising through registries or brokers for consumers to discover [10, 11].

\subsection{Service in NEC}

The U.K. MoD recognises that offering functionality is the main requirement in supporting military capability, and that functionality can be delivered without ownership of the delivery mechanism. In principle, suppliers are encouraged to respond to customers needs, providing the delivery of appropriate and up-to-date solutions into the military. Architectural characteristics required by NEC, such as flexible interoperability and future proof evolvability, can be in part provided by SOA, where organisations, systems and computing each have defined service interfaces.

In this paper, the definition of the term 'service' is not limited to Web Services and is not restricted to specific technologies. A service is an action performed by one entity on behalf of another and involves the transfer of value 
[12]. A formal service description is used to define a service and a service interface is used to access it. This level of abstraction allows the composition of services as shown in Figure 1.

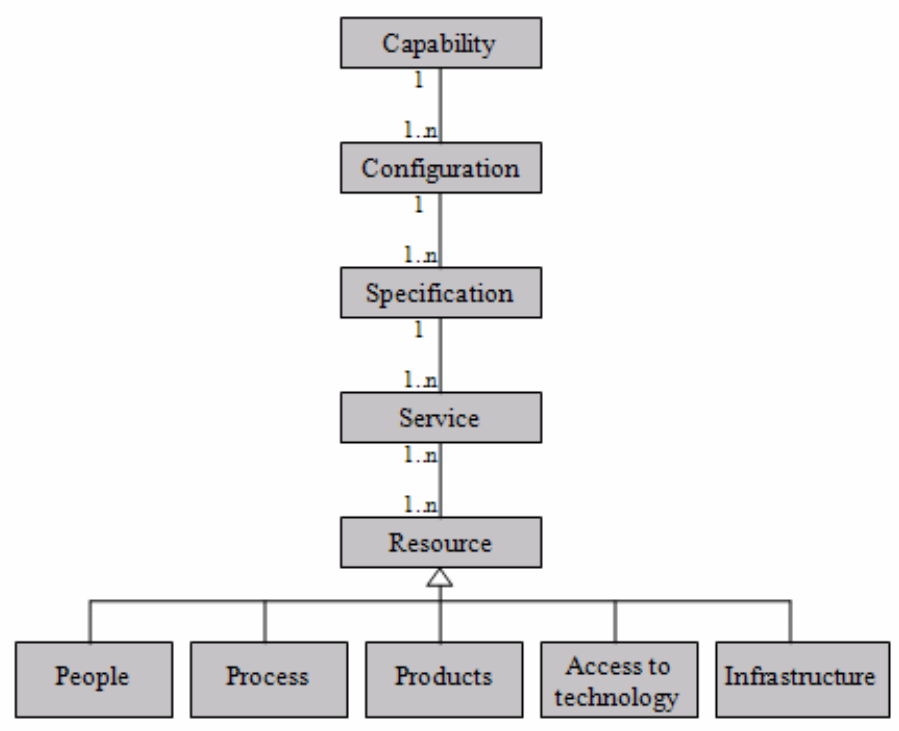

FIGURE 1: Capability concept model

A service description contains a functional element and non-functional element. The functional description defines the operation performed by the service. The non-functional description contains attributes of the Quality of Service (QoS), sub-divided into attributes about the service operation and attributes about the service provision. The operational attributes define the performance of the function, such as accuracy of the result and speed of the response. Attributes that describe the provision of a service include availability, security, cost and reliability.

\subsection{Advantages of SOA}

The Service-Oriented Architecture paradigm is concerned with the structure of service provision and consumption, and the infrastructure to support the interactions. Loose coupling [13] is the main benefit resulting from operating in this architecture for NEC. Loose coupling is obtained by abstracting the description of service provision from the implementation of service provision, thereby allowing different implementations to offer interchangeable services. This can then enable dynamic binding, where service implementations can be selected before service consumption by composing applications from suitable services based on their service descriptions.

Services are published and discovered in shared repositories, like Yellow Pages. Service descriptions contain the type of function provided, the location of the service and the QoS levels offered. QoS can be used to differentiate service implementations; alternatively other mechanisms such as reputation can be used to select services. QoS is assured by the service provider. The assured service may be a composite service that uses fault-tolerance means [14] such as redundancy to provide guarantees for availability [15] and using voting or process migration to protect against failure [16].

The architecture provides the ability to share services outside of traditional organisational boundaries. This is supported by mechanisms of discovery to match services during composition. The loosely coupled architecture allows services to be independent, and at the same time defined services interfaces allows the services to be 
interoperable [17]. Discovery mechanisms also promote the reuse of services in new applications and contexts not initially planned for, leading to lower development costs, lower opportunity costs and lower maintenance effort [11].

SOA also brings benefits of efficiency and managed consumption when changing from product delivery to a service delivery business model [18]. By comparison with product delivery, service delivery is a continuous process, assuring reliability by maintaining the service provision and evolving the service implementation to respond to changes in environment, situation, supply, information and ongoing development. These Product-Service Systems are defined as integrated product and service offering that deliver value in use. One example used in the DAME programme [7] is the Rolls-Royce Total Care Package (was Power-by-the-hour) for the lease of aircraft engines by commercial airlines.

\section{AGILITY IN NETWORKING}

In military systems, agility is an ability of the forces to adapt, to learn and to change to meet the threats that they face. Recent research studies (e.g. [19-21]) have revealed some similar properties between social networks and complex computer-based networks such as the Internet, the Web and peer-to-peer (P2P) networks, which have changed and evolved over time. In this section, complex networks are introduced, which provide essential base to further explore network enabling on complex military networks.

\subsection{Complex Networks}

From the users' point of view, the network exists to transfer of traffic (information or data) from one place to another. This is achieved through a number of nodes and links which form the network and algorithms which are used to direct the traffic across the network via the nodes and links. The user is interested in the Quality of Service of the network including the performance and dependability. These qualities are influenced by the design and operation of the network and the dynamic traffic passing through it. For small simple networks, such qualities are relatively easy to understand and calculate, but for large complex networks - such as those employed by the military, these qualities are difficult to assess. Two properties of complex networks are investigated in this section: small-world networks and scale-free networks, and then used to illustrate the resulting Qualities of Service.

\subsubsection{Small World Networks}

Small world phenomenon is a famous hypothesis in social networks that everyone in this world can be reached by a short chain of social acquaintances. In 1960s, the social psychologist Milgram conducted a famous small-world experiment [22]. He sent 60 letters to various recruits in Omaha, Nebraska who were asked to forward the letters to a stockbroker living at Massachusetts. The participants were only allowed to pass the letter by hand to friends who they thought might be able to reach the destination, no matter if directly or via a "friend of a friend". The most famous result of his experiment is that the average length of the resulting acquaintance chain is about six, which leads to the well-known phase "six degree of separation".

Working much more recently, Watts and Strogatz [23] presented a mathematical model to analyse the small world phenomenon. They explored small world networks by rewiring regular networks to introduce increasing amounts of disorder. From their analysis, these systems can be highly clustered, like regular lattices, and also have small characteristic path lengths, like random graphs. Following Watts's work, Kleinberg [24] discussed the navigation issue in small-world networks. He analysed small world networks by adding the long-range connections to a two-dimensional lattice. There is a decentralised algorithm with a specific parameter setting that achieves a very rapid delivery. However, for every other exponent, an asymptotically much larger delivery time is required. 


\subsubsection{Scale-free Networks}

Barabasi and Albert [25] mapped a part of the World Wide Web (WWW) in 1999 using a Web crawler and displayed the result as a graph. In this graph, all visited pages were represented as nodes and two pages are connected by a directed link if there is a web link between them. The surprising result is that the WWW did not have an even distribution of connectivity. In contrast, some nodes had many more connections than others. In this graph, The probability $p(k)$ that a node connected to $k$ other nodes was proportional to $k$ to the power of a constant $\gamma$ : $p(k) \propto k^{-\gamma}$. The similar phenomena have been also observed in some other networks, including some social and biological networks. These networks with the same characteristic are called scale-free networks (or power-law networks). Scale-free networks are also small world networks because they have large cluster coefficients and short average path lengths.

The factors leading to the scale-free distribution are complex. For example, a new node joining the network will have preference to early entrants; the longer a node has been in the network, the larger number of links to it; nodes with a larger number of links are easer to find; nodes with a higher capability (such as with large bandwidth and sharing a great deal of interesting content) also attracts more connections, which causes that the greater the capacity of the node, the faster its growth [26].

Barabasi and Albert [25] presented a "rich get richer" generative model called preferential attachment, where each new Web page creates links to existent Web pages with a probability distribution which is not uniform, but proportional to the current in-degree of Web pages. Thus, a page with many links will attract more links than a regular page, which generates a power-law, but their resulting graph still differs from the actual Web graph in other properties such as the presence of small tightly connected communities.

\subsection{Networking in NEC}

In the context of NEC, networking can be defined with regard to two aspects: network connectivity where every pair of distinct nodes is connected (without isolated sub-networks) and network awareness which enables each node to be aware of other nodes in the network.

The robustness of network connectivity is one of the most prominent strengths of scale-free networks. In random networks, a small number of random failures can collapse the network. In the scale-free networks, since the degree of connectivity is very heterogeneously distributed in the system, a random failure will very likely happen on one of the nodes with low degree of connectivity, which is most often not serious for the connectivity of the network. However, scale-free networks are vulnerable to intentional attacks on their hubs which are the nodes with high degree of connectivity. If the hubs of the system are attacked, the network is more rapidly fragmented than the corresponding random networks. Moreover, scale-free networks are also vulnerable to epidemics. In random networks, epidemics need to surpass a critical threshold (a number of nodes infected) before they spread to the whole network. The study in [27] indicates that the threshold for epidemics in scale-free networks is zero which makes the network vulnerable to epidemics.

A characteristic feature of small-world networks is the short diameter of network, which means there is always a very short path between any two nodes in the network. However, a node will not be aware of these short paths without global information. Neither Watts's model [23] nor Kleinberg's model [24] has given an efficient solution on how to use local information to discover other nodes in the network. lamnitchi, Ripeanu and Foster raised the open question in 
[28] about how to form and maintain inter-cluster connections and how to let nodes know which local nodes have external connections.

This issue was addressed in previous studies [29, 30], which presented a Small World Architecture for peer-to-peer Networks (SWAN) for resource discovery in a system with multiple agile groups. A semi-structured algorithm of SWAN is used to create and discover the long-range shortcuts between different groups, which can satisfy the following requirements of design:

(1) Not every node needs to connect to other groups;

(2) Each node needs to know or can easily find which nodes have external connections to which groups;

(3) External links to other groups need to be distributed within the group and cannot be centralised in one or a few nodes.

The importance of agility in networking has been recognised by both the U.S. and the U.K. militaries [31, 32]. Moffat and Atkinson discussed the relationships and effects of complex networks to US Department of Defense (DoD)'s Network Centric Warfare (NCW) and U.K. MoD's NEC in their books [32] and [31]. Moffat [32] argued that it is vitally important to consider warfare as a complex network that links and interacts with a surrounding much larger complex network with socio-economical and political context. A shift towards the creation of adaptive and complex networks of interaction will increase agility and increase the superiority of military forces over their adversaries [31].

Albert and Hayes proposed the famous "Power to the Edge" principle [33] in the Network Centric Warfare Framework (NCWF). They presented that the NCWF must support P2P relationships and information exchanges that transcend individual systems and organizations. In the NEC system, network nodes should be able to self-organise themselves into Edge Organisations on a non-hierarchical structure and form dynamic organisations with common interests and objectives. Edge Organisation (or Agile Organisation [31] defined for U.K. military) can generate higher flexibility and adaptability for military systems in responding to the new threats and environment encountered by the military in the future [33].

In this section, we have discussed agility in networking and how the need for effective peer-to peer communications has highlighted the benefits of small-world networks over scale-free networks in terms of vulnerability. As NEC is the integration of assets to fulfil the mission objective, networking is only part of the provision and different services need to be integrated across the network to deliver NEC. However, the rapid growth of information resources and services in military systems makes it difficult and challenging to manage dynamic information and resources efficiently. In the next section, service-oriented architecture for the provision of military capability will be analysed.

\section{SERVICE-ORIENTED ARCHITECTURE FOR THE PROVISION OF MILITARY CAPABILITY}

Using the definition adopted in NECTISE, services can be integrated to provide executable military capability [34]. Figure 2 illustrates agile integration of services for the delivery of military capability on SOA. In this architecture, each service provider provides a number of services, each service performs a set of functions, and some functions provided by services can be integrated to form a higher level of functionality to deliver a capability. For example, the BBC is a service provider which provides many services, such as news, weather, music and business. The weather service (a representation of the information service provided by the Met Office) includes a set of functions, such as weather conditions, temperature, wind, visibility, pressure, humidity and pollution. The weather conditions, wind and visibility function could be integrated with other functions from service providers to launch a high level of capability. 


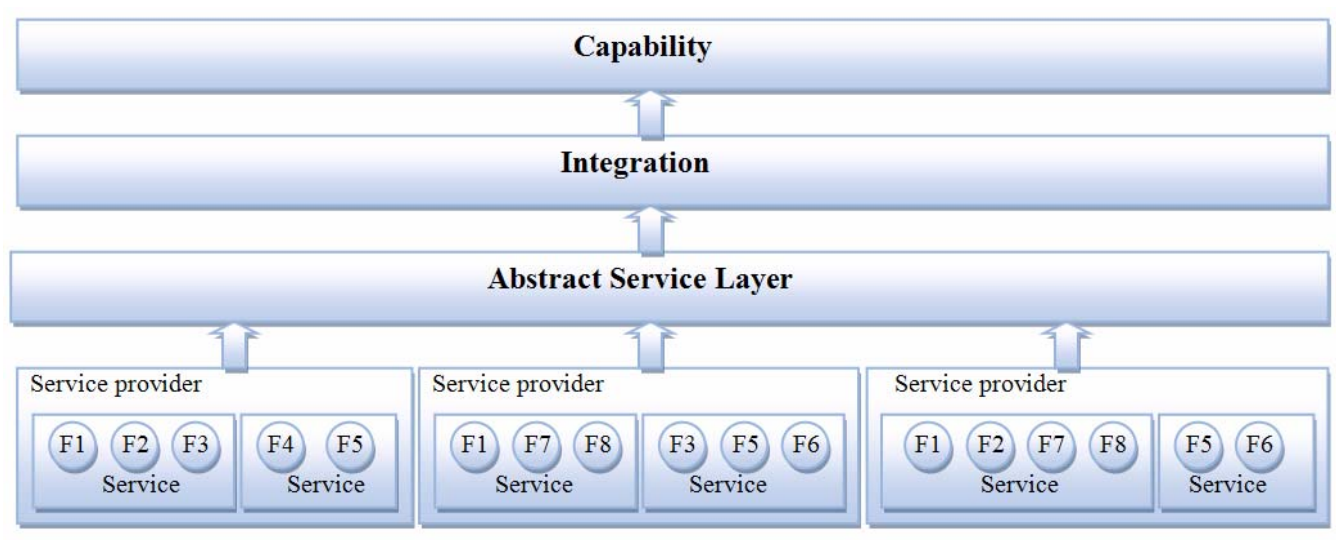

FIGURE 2: Service-oriented architecture for the delivery of military capability

\subsection{Motivations for Change}

NEC requires system integration of independent components that can evolve, operate in a dependable manner, managing system and component changes, be cost effective and connecting industrial, defence and pan-defence environments [34]. However, we live in a world where change is fundamental [3]. The motivations for evolution for NEC can be classified into two broad categories: external drivers and internal drivers. External drivers include:

- Military: to deploy capability to compete against an enemy's capability and improve that capability over time.

- Science and technology: to adapt to the technological changes. For example, changes in weapons: from cannon to rifled artillery to smart bombs [3].

- Commercial: to adapt to changes of sale agreement/contract; changes of budget; changes of cost (e.g. deployment and operation);

- Further drivers: to adapt to socio-political, legal, secure, cultural and religious issues.

- Internal drivers include:

- Maintenance: changes in the product maintenance cycles;

- Obsolescence: replacement of assets and components of products as they become unavailable;

- Economics: improving efficiency of delivery.

\subsection{Evolutionary Service-Oriented Architecture}

NEC is about the coherent integration of sensors, decision-makers, weapon systems and support capabilities to achieve the desired effect [3]. NEC requires Network Enabling by connectivity, information sharing and networking people, assets, and procedures, and the Capability to integrate them to fulfil mission objectives [10,35]. In order to deliver NEC, assets need to be integrated in context, to assist in human activity and provide dependable inter-operation [34]. To be successful in achieving this goal of NEC, evolutionary service-oriented architecture is a proper method to cope with unanticipated or unknown changes for the delivery of sustainable and dependable military capability.

In delivering capability, the SOA concept is applied to facilitate the matching of the needs of evolutionary architecture for NEC. SOA is designed for changes. As noted above, loose coupling is one of the key architectural principles of SOA, and this enables services to maintain a relationship that minimises dependencies and only requires maintaining an awareness of each other. Loose coupling is an approach where integration interfaces are developed with minimal assumptions between the sending/receiving parties, thus reducing the risk that a change in one service will force a change in another service [36]. 
The loose coupling of SOA enables service implementations to be inter-changed and modified. However, service integration is dependent on service interface definitions and requires management of workflow definitions to minimise impact on provision of military capability. In the architecture, fast paced changes are usually caused by evolution of services (e.g. adding or removing functions from services), evolution of service providers (e.g. adding and removing services by service providers), evolution of networks (e.g. network nodes joining and leaving the network) and evolution of capability (e.g. capability upgrade by integrating new functions). Changes in these cases could affect the dependability of provision of military capability. Capability could be lost if one of the subscribed services offering the requested functions is removed by the service provider, or one of the requested functions that was previously available on the subscribed service is removed or replaced by a different function. Service changes may lead to compatibility issues for the provision of a dependable and sustainable capability resulting from service interaction or interference.

To resolve these issues, an evolutionary service-based architecture is being addressed to adapt to or cope with these changes and unknowns for the reliable delivery of NEC in dynamic environments. Different from the specific system structures or lifecycle models, the evolutionary architecture for capability provides a framework for specification of generic configurations of military capability with across-platform attributes of services as shown in Figure 1. In the evolutionary service-based architecture, changes can be coped with by means of either re-configuration of existing services or integration of new services into the system. To minimise service disruption and to enable service enhancement, runtime assessment is performed to measure the impact of continuous evolution resulting from changes within the NECTISE evaluation framework by means of both Measures of Effectiveness (MoE) and Measures of Performance (MoP), as noted in Section 4.3 below.

\subsection{Architecture Evaluation}

The NECTISE evaluation framework is an important part of the evolutionary service-based architecture. In order to understand how to achieve service integration for military capability, this work is investigating ways of evaluating capabilities composed of configurations of services using Measures of Effectiveness as a basis for evaluating capability against scenarios, rather than attempting to evaluate specific architectures directly. Since the solution evaluates capabilities rather than specific architectures, it will be applicable to whichever architectural model is chosen within the NECTISE project and it should have a wide applicability outside the scope of the NECTISE project.

In this framework, the impact of changes in services and capability is measured in both effectiveness and performance as shown in Figure 3. Measures of Performance are used to validate implementations of service descriptions, whilst Measures of Effectiveness are used to assess a configuration of services composed to satisfy a capability. Measures of Effectiveness are applied using a composition of service descriptions and can therefore be applied at a conceptual level, independent of the services consumed [34]. 


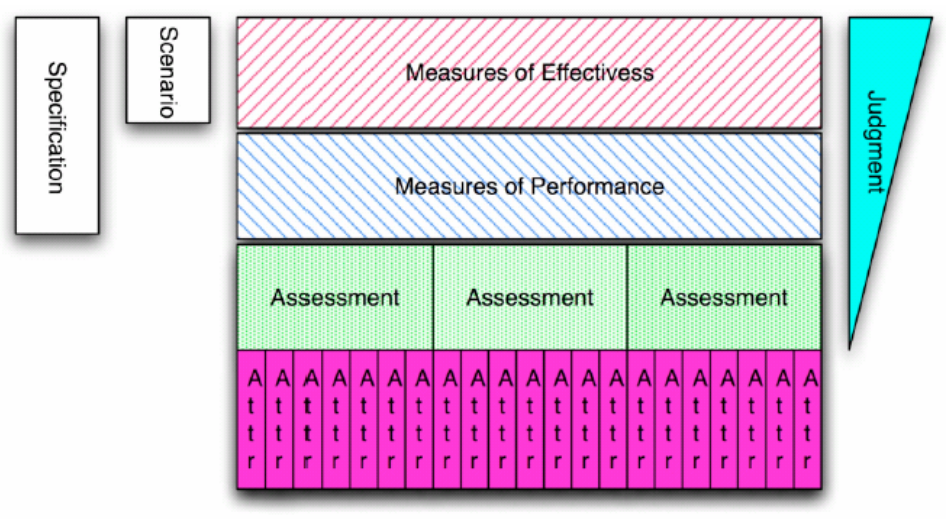

FIGURE 3: Structure of measures

Measures of Performance can be made up of a number of assessments about Cost of Service (CoS) and QoS, drawing on different assessment methods to feed into the judgmental rules applied within the Measures of Performance. CoS for each configuration of capability needs to be measured to optimise the resources for the provision of military capability. Quality of Service is one of the most important factors to deliver a dependable and sustainable capability. Current QoS descriptions of services need to be extended for the context of NEC, by investigating those attributes of capability that can be characterised and measured for integrated services.

In this framework, an assessment is based on a set of method specific rules and a set of attributes of CoS and QoS. The attributes include:

- Availability - the probability that a service is present and ready for use;

- Reliability - the capability of maintaining the service and service quality;

- Safety - the absence of catastrophic consequences;

- Confidentiality - information is accessible only to those authorised to use it;

- Integrity - the absence of improper system alterations;

- Maintainability - to undergo modifications and repairs;

- Cost - the price customers are willing to incur to obtain a level of service.

Implicit in this model is the concept of judgment. Judgmental evaluations are small at the base of the model where explicit measurements are made but this increases as the measurements are combined into assessments, which form the basis of MoP and increases to a maximum towards the top of the model, where scenario based MoE are obtained.

\section{CONCLUSION AND FUTURE WORK}

Enhanced military effect can be achieved through dynamic networking of people, assets and procedures and seamless integration of them to fulfil mission objectives. From the above discussion, it can be concluded that Service-Oriented Architecture should be able to provide the agility to handle the change and dynamic evolution needed in NEC. In this paper, agility in networking and system integration has been discussed for the provision of dependable and sustainable military capability. The evaluation framework has been presented in which the impact of changes can be measured in both effectiveness and performance. 
This paper reports "work in progress" and the essential base for future research in NECTISE. In future work, instances of the framework will be evaluated in depth by modelling and simulations to show the dependability of military capability in a dynamic environment. Agile models for service integration, service discovery and service development will be further investigated for the provision of dependable and sustainable military capabilities. The investigation will link to lifecycles for service delivery and agile methods to respond to changes owing to, for example, faults, customer need, technology developments and obsolescence. The next step will be to investigate the relationships among four themes of NEC readiness - agility, availability, dependability and affordability - in terms of how to use agility to achieve better dependability of the provision of capability even with the services with low availability, and minimising the impact on the affordability of capability, through the use of both simulation-based and realistic evaluations in order to efficiently reuse the existing resources to achieve the optimised military capability in a dynamic environment.

\section{ACKNOWLEDGEMENT}

The work reported in this paper has been supported by the NECTISE programme jointly funded by BAE Systems and the U.K. Engineering and Physical Sciences Research Council Grant EP/D505461/1.

\section{REFERENCES}

[1] ALBERTS, D. S., GARSTKA, J. J. \& STEIN, F. P. (1999) Network Centric Warfare: Developing and Leveraging Information Superiority, CCRP.

[2] LAMBERT, D. A. (1999) Ubiquitous Command And Control. International Conference on Information, Decision and Control. Adelaide, Australia.

[3] UK Ministry of Defence, Joint Services Publication 777, Edition 1, (2005) p. 1,

[4] Network Enabled Capability Though Innovative System Engineering (NECTISE) Project Website Available: http://www.seic-loughborough.com/AllPages.html?Page=350

[5] Network Enabled Capability: an Introduction, Version 1.0 (April 2004), Ministry of Defense, U.K.

[6] UK Ministry of Defence, Defence Industrial Strategy: Defence White Paper (CM6697) (2005).

[7] RUSSELL, D. J., DEW, P. M. \& DJEMAME, K. (2007) A secure service-based collaborative workflow system. International Journal of Business Process Integration and Management, 2, 230-244.

[8] TURNER, M., BUDGEN, D. \& BRERETON, P. (2003) Turning software into a service. IEEE Computer, 36, 38-44.

[9] SMITH, H. \& FINGAR, P. (2003) Business Process Management: The Third Wave, Tampa, FL., Meghan-Kiffer Press.

[10] RUSSELL, D. \& XU, J. (2007) Service Oriented Architectures in the Provision of Military Capability. UK e-Science All Hands Meeting.

[11] ALONSO, G., CASATI, F., KUNO, H. \& MACHIRAJU, V. (2004) Web Services - Concepts, Architecture and Applications, London, Springer.

[12] O'SULLIVAN, J., EDMOND, D. \& TER HOFSTEDE, A. H. M. (2003) Service Description: A survey of the general nature of services. Queensland University of Technology.

[13]PAPAZOGLOU, M. P. \& DUBRAY, J.-J. (2004) A Survey of Web Service Technologies. University of Trento, Italy.

[14] AVIZIENIS, A., LAPRIE, J.-C. \& RANDELL, B. Fundamental Concepts of Dependability (2001) UK Newcastle University Report no. CS-TR-739. 
[15] CRAWFORD, C. H., BATE, G. P., CHERBAKOV, L., HOLLEY, K. L. \& TSOCANOS, C. (2005) Toward an on demand service-oriented architecture. IBM Systems Journal, 44, 81-107.

[16] TOWNEND, P. \& XU, J. (2006) Topology-aware fault-tolerance in service-oriented grids. IN COX, S. J. (Ed.) Processdings of the UK e-Science All Hands Meeting 2006. Nottingham, UK, NeSC.

[17]WS-I Organization, Available: http://www.ws-i.org/

[18]MONT, O. K. (2002) Clarifying the concept of product-service system. Journal of Cleaner Production, 10, 237-245.

[19] HONG, T. (2001) Chapter Fourteen: Performance. IN ORAM, A. (Ed.) Peer-to-Peer: Harnessing the Power of Disruptive Technologies. O'Reilly.

[20] SAROIU, S., GUMMADI, P. K. \& GRIBBLE, S. D. (2002) A Measurement Study of Peer-to-Peer File Sharing Systems. International Conference on Multimedia Networking and Computing. Santa Barbara, CA.

[21] GUMMADI, K. P., DUNN, R. J., SAROIU, S., GRIBBLE, S. D., LEVY, H. M. \& ZAHORJAN, J. (2003) Measurement, Modelling and Analysis of a P2P File-sharing Workload. ACM Symposium on Operating Systems Principles. Bolton Landing, New York.

[22] MILGRAM, S. (1967) The Small World Problem. Psychology Today, 2, 60-67.

[23]WATTS, D. \& STROGATZ, S. H. (1998) Collective Dynamics of Small-World Networks. Nature 393, 440-442.

[24] KLEINBERG, J. (2000) Navigation in a Small World. Nature, 406, 845.

[25] BARABASI, A. L. \& ALBERT, R. (1999) Emergence of Scaling in Random Networks. Science, 286, 509-512.

[26]ROBB, J. Scale-free Networks, Global Guerrillas (May 2004), Available:

http://globalguerrillas.typepad.com/globalguerrillas/2004/05/scalefree_terro.html

[27]BOGUNA, M., ROMUALDO PASTOR-SATORRAS \& VESPIGNANI, A. (2003) Absence of Epidemic Threshold in Scale-Free Networks with Degree Correlations. Physical Review Letters, 90, 028701-1 - 028701-4.

[28] IAMNITCHI, A., RIPEANU, M. \& FOSTER, I. (2002) Locating Data in Peer-to-Peer Scientific Collaborations. International Workshop on Peer-to-Peer Systems. Cambridge, USA.

[29] LIU, L., ANTONOPOULOS, N. \& MACKIN, S. (2007) Small World Peer-to-peer for Resource Discovery. International Conference on Information Networking, Lecture Notes in Computer Science. Estoril, Portugal.

[30] LIU, L., ANTONOPOULOS, N. \& MACKIN, S. (2007) Fault-tolerant Peer-to-Peer Search on Small-World Networks. Journal of Future Generation Computer Systems, 23, 921-931.

[31]ATKINSON, S. R. \& MOFFAT, J. (2005) The Agile Organization, Command and Control Research Program (CCRP) Publications Series.

[32] MOFFAT, J. (2003) Complexity Theory and Network Centric Warfare, Command and Control Research Program (CCRP) Publications Series.

[33] ALBERTS, D. S. \& HAYES, R. E. (2005) Power to the Edge, Command and Control Research Program (CCRP) Publications Series.

[34] RUSSELL, D., LOOKER, N., LIU, L. \& XU, J. (2008) Service-Oriented Integration of Systems for Military Capability. IEEE International Symposium on Object/component/service-oriented Real-time distributed Computing, in press. Orlando, Florida

[35] RUSSELL, D., LOOKER, N. \& XU, J. (2006) SOA, Dependability, and Measures and Metrics for NEC. IET Forum on Capability Engineering.

[36] Loose coupling, Wikipedia, Available: http://en.wikipedia.org/wiki/Loose_coupling 